\title{
O PROCEDIMENTO DE CONSTRUÇÃO DAS VARETAS DO PROMPTUARIO DE JOHN NAPIER (1550-1617)
}

\author{
THE CONSTRUCTION PROCEDURE OF STRIPS FROM \\ JOHN NAPIER'S (1550- 1617) PROMPTUARY
}

\author{
Pedro Henrique Sales Ribeiro ${ }^{1}$; Davi Souza Cavalcante ${ }^{2}$; Ana Carolina Costa Pereira ${ }^{3}$
}

\begin{abstract}
RESUMO
Ao longo de períodos históricos, o desenvolvimento do conhecimento matemático se deu, entre outros motivos, a partir das carências de determinados povos e culturas. Um dos momentos históricos em que isso ocorreu foi na Idade Moderna, que, ao romper com os padrões anteriores, impôs novas necessidades, incluindo, tantas outras, a de se conseguir calcular de forma mais ágil e eficaz. Assim, durante a revolução científica, diversos tratados que continham instrumentos matemáticos focados em resolver essa questão, foram publicados. Entre estes tratados, podemos destacar o Rabdologiae, Seu Numerationis Per virgulas ..., publicado em 1617 pelo matemático escocês John Napier, e que aborda três instrumentos para cálculos, incluindo o Promptuario. Dessa forma, esse artigo tem o objetivo de apresentar o processo realizado pelo autor, para a construção física de uma parte importante deste aparato, e para esse propósito, nos valemos de uma metodologia qualitativa adjunta a uma pesquisa documental, já que utilizamos um documento original, em sua versão traduzida para o inglês por William Frank Richardson em 1990 e que está contida no livro A Vida e as Obras de John Napier, publicado no ano de 2017. Portanto, após compreender o procedimento de construção do instrumento, e reconhecer os aspectos e conhecimentos matemáticos, especialmente geométricos, que são mobilizados, concluímos que é possível incorporar essa operação em uma proposta de interface entre ensino e história da matemática.
\end{abstract}

Palavras-chave: Promptuario; Instrumentos Matemáticos; História da Matemática; John Napier.

\footnotetext{
${ }^{1}$ Graduando em Licenciatura em Matemática - Universidade Estadual do Ceará (UECE). Bolsista de Iniciação Científica - Universidade Estadual do Ceará (UECE), Fortaleza, Ceará, Brasil. Endereço para correspondência: Rua São Francisco, 114, Pajuçara, Maracanaú, Ceará, Brasil, CEP: 61932-500. E-mail: henrique.ribeiro@aluno.uece.br.

ORCID iD: https://orcid.org/0000-0001-9270-5339.

${ }^{2}$ Graduando em Licenciatura em Matemática pela Universidade Estadual do Ceará (UECE). Bolsista de Iniciação Científica - Universidade Estadual do Ceará (UECE), Fortaleza, Ceará, Brasil. Endereço para correspondência: Rua Ribeiro da Silva., 700, A-Altos, Monte Castelo, Fortaleza, Ceará, Brasil, CEP: 60325-210. E-mail: davi.cavalcante@aluno.uece.br.

(D) ORCID iD: https://orcid.org/0000-0003-2638-5896

${ }^{3}$ Doutora em Educação pela Universidade Federal do Rio Grande do Norte (UFRN). Professora Adjunta Universidade Estadual do Ceará (UECE) e coordenadora do curso de Licenciatura em Matemática, Fortaleza, Ceará, Brasil. Endereço para correspondência: Av. Silas Munguba, 1700 - Campus do Itaperi, Serrinha, Fortaleza, Ceará, Brasil, CEP: 60740-903. E-mail: carolina.pereira@uece.br.
} 
Pedro Henrique Sales Ribeiro, Davi Souza Cavalcante, Ana Carolina Costa Pereira

O procedimento de construção do Promptuario de John Napier (1550-1617)

\begin{abstract}
Throughout historical periods, the development of mathematical knowledge occurred, among other reasons, from the needs of certain peoples and cultures. One of the historical moments in which this occurred was in the Modern Age, which, by breaking with previous standards, imposed new needs, including, many others, that of being able to calculate in a more agile and effective way. Thus, during the scientific revolution, several treaties that contained mathematical instruments focused on solving this issue were published. Among these treaties, we can highlight the Rabdologiae, Seu Numerationis Per virgulas ..., published in 1617 by the Scottish mathematician John Napier, and which addresses three instruments for calculations, including the Promptuari. Thus, this article aims to present the process carried out by the author, for the physical construction of an important part of this apparatus, and for that purpose, we use a qualitative methodology attached to a documentary research, since we use an original document, in its version translated into English by William Frank Richardson in 1990 and which is contained in the book The Life and Works of John Napier, published in the year 2017. Therefore, after understanding the instrument construction procedure, and recognizing the mathematical aspects and knowledge, especially geometric, that are mobilized, we conclude that it is possible to incorporate this operation in a proposal for an interface between teaching and the history of mathematics.
\end{abstract}

Keywords: Promptuary; Mathematical Instruments; History of Mathematics; John Napier.

\title{
Introdução
}

Sendo, por muitas vezes, classificada como um "Ciência Exata", a Matemática tende a ser encarada somente como um conhecimento finalizado e concreto. No entanto, esta definição pode falhar quando visualizamos, de uma maneira mais ampla, a forma na qual ela foi construída ao longo de diversos períodos históricos, e assim, podemos afirmar que "a Matemática é uma ciência humana, fruto das necessidades e preocupações de diferentes culturas, em diferentes momentos históricos” (BRASIL, 2018).

Considerando que a Matemática não é algo puramente concreto e acabado, então também não podemos afirmar que sua história seja, e portanto,

[...] devemos tomar o cuidado de não reduzir a história da matemática a dados biográficos ou a uma coleção de curiosidades. Tampouco a história da matemática pode ser considerada um repositório de informações fixas, como se fosse um conjunto de ideias ultrapassadas e antigas. (SAITO, 2015, p. 20).

Dessa forma, partindo destas duas concepções, é possível afirmar que os conhecimentos matemáticos desenvolvidos e construídos em um determinado período histórico, derivaram não só de motivações pessoais, mas principalmente das carências que estavam sendo enfrentadas no contexto em questão.

Como caso particular, podemos citar o período da Idade Moderna, que se iniciou no século XV, mais precisamente no ano de 1453, e que se mostrou adversa ao momento vivido anteriormente, a Idade Média. Neste contexto de mudança, diversas questões de 
Pedro Henrique Sales Ribeiro, Davi Souza Cavalcante, Ana Carolina Costa Pereira

O procedimento de construção do Promptuario de John Napier (1550-1617)

ordem política, social, econômica, científica e até mesmo cultural, vieram à tona, juntas ao movimento que hoje conhecemos por Renascimento.

Em conjunturas posteriores, estas questões ganharam destaques individuais, e foram motivos de grandes transformações. Um destes destaques foi a Revolução Cientifica, que teve início no século XVI, e trouxe consigo um grande marco histórico, a "popularização", no sentindo de facilitação, da imprensa e uma consequente disseminação de diversos tratados de cunho científico.

Ao analisar alguns destes tratados, é possível encontrar aqueles nos quais estão contidos os chamados "instrumentos" que

entraram em uso para facilitar a resolução de problemas matemáticos e investigar a natureza por meios de recursos observacionais e experimentais. Dentre esses instrumentos, encontramos aqueles denominados "matemáticos", isto é, instrumentos que foram concebidos para medir aquilo que Aristóteles denominava "quantidades" (distâncias e ângulos). (SAITO, 2015, p. 187)

Entre as mais variadas obras que foram publicadas neste período, e que abordavam instrumentos matemáticos, podemos destacar o tratado Rabdologiae, seu Numerationis per Virgulas libri duo: Cum Appendice de expeditissimo Multiplicationis promptuario. Quibus accessit \& Arithmeticae Localis Liber unus. ${ }^{4}$, publicado no ano de 1617, na cidade de Edimburgo, e de autoria do inventor e matemático escocês John Napier (1550-1617) 5 .

Nesta publicação, o autor se dedica a expor três destes instrumentos, que são destinados para cálculos algébricos, e nas palavras do próprio,

\footnotetext{
O primeiro deles, o qual chamo de Rabdologia, utiliza barras com números inscritos nelas. O segundo, que para a multiplicação é o mais rápido de todos, usa varetas dispostas em uma caixa; para esse, o nome Promptuario para multiplicação não vai ser inadequado. O terceiro e último usa a Aritmética de localização e é executado em um Tabuleiro de Xadrez. (NAPIER, 2017, p. 653, tradução nossa) ${ }^{6}$
}

4 A partir de agora, por questões de facilidade, nos referiremos apenas como Rabdologiae, Seu Numerationis per Virgulas....

${ }^{5}$ Para maiores informações sobre a vida de John Napier e sua obra Rabdologia, vide: Martins e Pereira (2018, 2019),

${ }^{6}$ Em inglês, lê-se: "The first of these, which I call Rabdology, uses rods with numbers on them. The second, which for multiplication is the fastest of all, uses strips arranged on a box; for this the name promptuary for Multiplication will not be inappropriate. The third and last uses location Arithmetic and is performed on a chess-board" (NAPIER, 2017, p. 653) 
Embora a obra aborde estes três importantes instrumentos matemáticos, este estudo está restringido somente ao Promptuario $^{7}$, e neste caso específico, está também limitado ao seu procedimento de construção, que foi exposto por Napier em um apêndice de dezessete páginas, e que está localizado logo após os dois primeiros livros do tratado.

Dessa forma, nesse artigo iremos apresentar o processo realizado por John Napier para a construção física de uma parte importante do Promptuario, contido em seu tratado, Rabdologiae, Seu Numerationis Per virgulas ..., publicado em 1617.

\section{Metodologia}

Por se tratar de uma análise realizada a partir de um texto original ${ }^{8}$, optamos por desenvolver este estudo amparado por uma metodologia qualitativa, de cunho descritivo, associada a um processo de pesquisa documental, uma vez que as informações aqui postas, foram retiradas estritamente de um documento, tendo o objetivo de compreender a forma como este procedimento de construção está disposto e sua possível contribuição para o ensino de matemática (KRIPKA, SCHELLER E BONOTTO, 2015).

É importante ressaltar que o documento escolhido não é todo o tratado Rabdologiae, Seu Numerationis per Virgulas, mas somente um excerto deste, precisamente, o apêndice denominado "Promptuario de alta velocidade para multiplicação", que está contido entre as páginas 85 a 102, em sua versão publicada no ano de 1617 e no idioma latim.

Apesar desta publicação ser a primeira versão, optamos, prezando maior facilidade na compreensão do idioma, por utilizar a tradução em inglês, que foi realizada por William Frank Richardson no ano de 1990, e que se encontra também publicada no livro The Life and Works of John Napier, de autoria dos pesquisadores Brian Rice, Enrique González-Velasco e Alexander Corrigan, e publicada em 2017. Neste livro, o excerto escolhido se encontra entre as páginas 713 a 726.

Embora tenhamos utilizado uma tradução da obra, iremos considerá-la, para efeitos de classificação metodológica, como um texto original, uma vez que "para o educador matemático, é possível considerar um documento original traduzido, dependendo da sua intencionalidade" (SILVA, 2018, p. 40).

\footnotetext{
${ }^{7}$ Para manter a fidelidade ao texto, optamos por manter o nome do instrumento escrito em latim, e não utilizar a versão "Prontuário", existente na língua portuguesa.

${ }^{8}$ Vide: Silva (2018)
} 
Pedro Henrique Sales Ribeiro, Davi Souza Cavalcante, Ana Carolina Costa Pereira

O procedimento de construção do Promptuario de John Napier (1550-1617)

\section{O Promptuario}

Sendo a última de todas as descobertas do autor (NAPIER, 2017), o Promptuario é um conjunto (Figura 1), composto por varetas, sendo elas verticais, contendo os múltiplos dos dez algarismos, e horizontais, que são perfuradas em determinados espaços, bem como por uma caixa, que serve tanto para guardar estas varetas, como para orientar a correta disposição delas e a consequente realização dos cálculos .

\section{Figura 1 - O Conjunto que compõe o instrumento Promptuario}

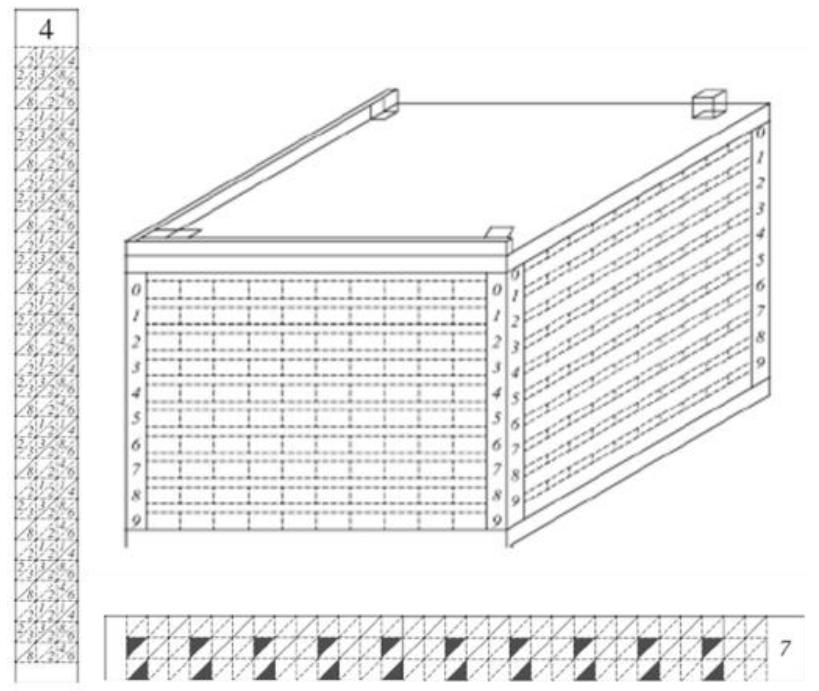

Fonte: Napier (2017, p. 714, 715, 719)

Segundo o próprio autor, com a utilização desse instrumento, todas as multiplicações podem ser facilmente realizadas, independente do seu tamanho (NAPIER, 2017), e na concepção de Rice; González-Velasco; Corrigan (217, p.40, tradução nossa), ele é

provavelmente o mais poderoso dispositivo de Napier, [...] e a máquina envolve uma versão mais complexa dos Ossos de Napier, permitindo que a resposta de uma multiplicação seja visualizada através das aberturas, uma vez que o número a ser multiplicado foi disposto nas varetas verticais, e o multiplicador disposto em varetas horizontais 9 .

Portanto, tendo em vista a opinião de Napier (2017) sobre sua própria criação, e o pensamento dos autores, é possível perceber a importância dessa invenção, devido a sua capacidade de efetuar longos cálculos de multiplicação sem grandes dificuldades.

\footnotetext{
${ }^{9}$ Em inglês, lê-se: “probabily the most powerful of Napier's devices [...] and the machine involves a more complex version of Napier's Bones, allowing the answer to a multiplication to be read through apertures once the number to be multiplied has been set out in vertical strips and the multiplier set out in horizontal strips." (RICE, GONZÁLEZ-VELASCO, CORRIGAN, 2017, p.40)"
} 
Pedro Henrique Sales Ribeiro, Davi Souza Cavalcante, Ana Carolina Costa Pereira

O procedimento de construção do Promptuario de John Napier (1550-1617)

\section{O início da construção}

Em um primeiro momento, o autor determina o material na qual as varetas devem ser construídas, ele ressalta que elas "devem ser feitas de marfim ou qualquer outro material branco sólido" (NAPIER, 2017, p. 713, tradução nossa) ${ }^{10}$, esta determinação provavelmente se dá pela rigidez que se faz necessária para uma correta manipulação do instrumento, uma vez que, se muito maleável, pode haver dificuldades para manter as varetas na posição definida pelo autor, e consequentemente interferindo na precisão dos cálculos.

Em seguida, Napier passa a explicar como definiu as quantidades de varetas que confeccionou, segundo ele, "para multiplicar números inferiores a 100000, você precisará de cem delas e mais (ou menos) proporcionalmente ao tamanho dos números que deseja multiplicar" (NAPIER, 2017, p. 713, tradução nossa) ${ }^{11}$. Essa exigência é feita porque existem um total de vinte varetas distintas, sendo dez delas verticais e dez horizontais, logo para um número de até cinco algarismos (ou seja, até 99999), serão necessárias cinco de cada uma das varetas distintas, portanto 5 x 20 varetas, o que resulta nas cem varetas explicitadas pelo autor. Além disso, ele afirma ter escolhido construir 200 varetas, e assim podendo multiplicar números até 10000000000. (NAPIER, 2017)

Posteriormente, é abordado o tamanho destas varetas, que devem ter onze dedos de comprimento, que atualmente, essa unidade de medida corresponde a 20,9 cm, e um dedo de largura, ou seja, 1,9 cm (NAPIER, 2017). Seguindo, o autor apresenta as primeiras as marcações a serem realizadas, que estão diretamente ligadas a este tamanho das varetas.

Neste momento, é necessário que se faça duas divisões nas extremidades, a primeira irá definir a margem maior, que deverá ter $2 / 3$ de um dedo, e a segunda irá delimitar a margem menor, que deverá ser de 1/3 de um dedo, sobrando assim, dez dedos de comprimento, que deverão ser divididos em dez quadrados (NAPIER, 2017), estes quadrados terão dimensões 1 dedo por 1 dedo, já que a largura já havia sido determina.

Após estas primeiras marcações, Napier afirma que metade das varetas que você construir "devem ter um quarto de dedo [de espessura]; as outras [...] devem ter apenas metade da espessura dessa (mas mais se o material exigir)" (NAPIER, 2017, p. 713,

\footnotetext{
${ }^{10}$ Em inglês, lê-se: "should be made of ivory or any other solid white material” (NAPIER, 2017, p.713)

${ }^{11}$ Em inglês, lê-se: "For multiplying numbers less than 100000, you will require one hundred of them, and more (or less) in proportion to the size of the numbers you wish to multiply" (NAPIER, 2017, p. 713)
} 
Pedro Henrique Sales Ribeiro, Davi Souza Cavalcante, Ana Carolina Costa Pereira

O procedimento de construção do Promptuario de John Napier (1550-1617)

tradução nossa $)^{12}$, esta diferenciação entre as espessuras é utilizada para distinguir a construção das varetas verticais das horizontais, da seguinte maneira,

Cada uma das [...] varetas mais grossas devem ser colocadas na sua frente, de modo que apontem para o seu peito com a margem maior mais longe de você e com a margem menor mais perto. Essas varetas eu chamo de varetas verticais. Cada das varetas mais finas, por outro lado, deve ficar com a margem mais larga à direita e a mais estreita à esquerda, em outras palavras, perpendicularmente aos demais. Essas varetas, portanto, chamo de varetas horizontais. (NAPIER, 2017, p. 713, tradução nossa). ${ }^{13}$

Assim, quando todas as varetas estiverem dispostas desta maneira, é possível prosseguir com o procedimento de construção. É importante ressaltar que as próximas marcações serão realizadas em todos os dez quadrados, que foram formados a partir da divisão inicial exposta anteriormente.

\section{Os dez quadrados}

Inicialmente, o autor afirma que é necessário que se desenhe uma linha diagonal, que não deve ser apagável, em cada um dos dez quadrados, essas diagonais devem ser “do ângulo inferior esquerdo para o ângulo superior direito" (NAPIER, 2017, p. 714, tradução nossa) ${ }^{14}$, ao se realizar esse procedimento, podemos perceber que cada um dos dez quadrados, foi divido agora em dois triângulos isósceles. (NAPIER, 2017).

As próximas marcações envolvem conhecimentos geométricos mais sofisticados, uma vez que se faz preciso a divisão de um segmento em três partes iguais. Essa divisão é realizada em cada lado, de cada um dos dez quadrados, e após isto, Napier (2017, p. 714, tradução nossa) ${ }^{15}$, afirma ser necessário que se “desenhe linhas [apagáveis] através os pontos opostos de divisão", ou seja, que se trace o segmento contendo estes pontos, e que este segmento seja perpendicular ao lado do quadrado.

\footnotetext{
${ }^{12}$ Em inglês, lê-se: "should be one quarter of a finger thick; the other [...] should be only half as thick as that (but more if the material requires it)" (NAPIER, 2017, p. 713)

${ }^{13}$ Em inglês, lê-se: "Each of the [...]thicker strips is to be placed before you in such a way that it points toward your chest with the greater margin farther from you and the lesser margin closer. These strips I call vertical strips. Each of the thinner strips, on the other hand, should lie with the wider margin to the right and the narrower to the left, in other words, at right angles to the others. These strips I therefore call horizontal strips."

${ }^{14}$ Em inglês, lê-se: "from the lower left to the upper right angle" (NAPIER, 2017, p. 714)

${ }^{15}$ Em inglês, lê-se: "draw lines through the opposite points of division" (NAPIER, 2017, p. 714)
} 
Pedro Henrique Sales Ribeiro, Davi Souza Cavalcante, Ana Carolina Costa Pereira

O procedimento de construção do Promptuario de John Napier (1550-1617)

Ao realizar a marcação anterior, se torna possível perceber que cada um dos dez quadrados iniciais foi dividido agora em nove quadrados menores (Figura 2), com uma dimensão de $1 / 3$ de dedo por $1 / 3$ de dedo.

Figura 2 - Processo de marcação de cada um dos dez quadrados.

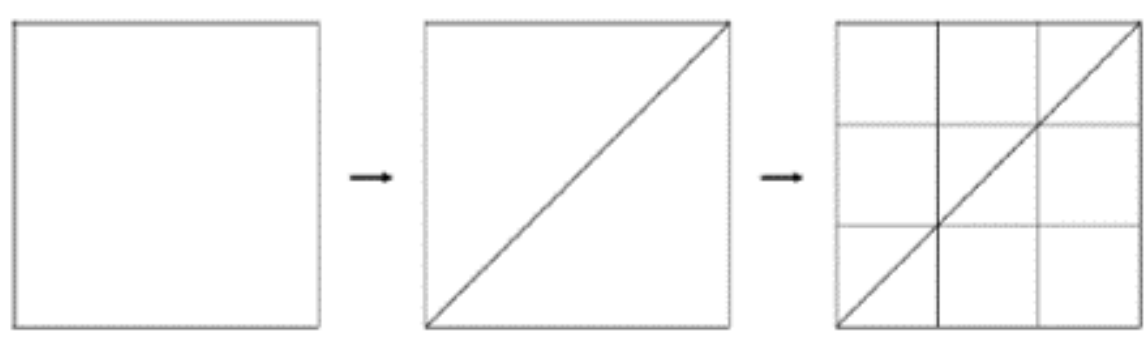

Fonte: Elaborado pelos autores;

Por fim, deve-se reproduzir a primeira marcação realizada, a de traçar a diagonal, porém agora de forma apagável, e em cada um dos quadrados pequenos (NAPIER, 2017). É importante, como ressalta o autor, que as diagonais traçadas nesse momento seja paralela a primeira diagonal, já que, caso contrário, os quadrados pequenos que já foram cortados anteriormente pela diagonal maior, seriam agora novamente cortados, sendo divididos em 4 partes, resultado em 24 triângulos em cada um dos quadrados maiores, o que contraria a indicação seguinte do autor.

Ao traçar corretamente as diagonais, cada um dos dez quadrados inicias, estarão divididos em 18 triângulos, "que eu chamarei de lugares a partir de agora" (NAPIER, 2017, p. 714, tradução nossa) ${ }^{16}$, e em cada um destes lugares, o autor realiza a inscrição, de maneira apagável, das nove letras no alfabeto, conforme a Figura 3.

Figura 3 - As nove letras em seus respectivos "lugares"

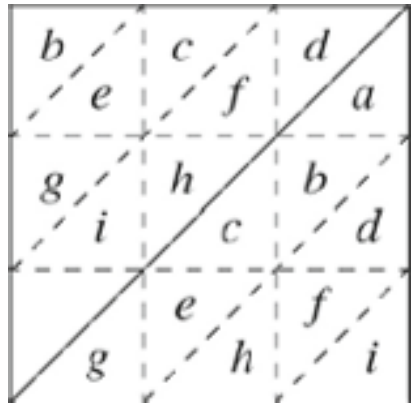

Fonte: Napier (2017. p.714).

${ }^{16}$ Em inglês, lê-se: “which I will henceforth call places” (NAPIER, 2017, p. 714) 
Resta agora apagar as linhas que foram consideradas pelo autor como "apagáveis", e após isso "um dos dígitos deve ser gravado ou entalhado na margem maior de cada vareta” (NAPIER, 2017, p. 714, tradução nossa) ${ }^{17}$, ou seja, é necessário identificar cada uma das varetas, utilizando os dez algarismo, de forma que, caso seja construída 200 varetas (Como no caso do autor),

\begin{abstract}
Em dez das cem varetas mais grossas e dez das mais finas, escreva (indelevelmente) a cifra 0 . Em outras dez das mais grossas e o mesmo número das mais finas, escreva (indelevelmente) o algarismo 1. Em outras dez das mais grossas e o mesmo número das mais finas, escreva (indelevelmente) o algarismo 2. Em outras dez das mais grossas e outras dez das mais finas, escreva na margem maior o algarismo 3. E assim por diante, quatro, cinco e o restante até nove inclusive, até que todas as duzentas varetas tenham um algarismo escrito em sua margem maior.
\end{abstract}

Até o momento, todas as marcações foram comuns a ambas as varetas, porém, neste ponto, todas estão fisicamente construídas e prontas para serem graduadas, então, o autor passa a diferenciar o processo de graduação delas, que será abordado em publicações futuras.

\title{
Considerações Finais
}

Com base na pesquisa documental realizada, foi possível compreender a forma como o procedimento de construção das varetas do Promptuario está apresentado dentro do texto escolhido, bem como reconhecer os aspectos matemáticos, principalmente de ordem geométrica, que são mobilizados durante todo o processo.

Além disso, conseguimos entender a motivação, tanto estrutural quanto matemática, de determinadas exigências e definições estabelecidas pelo autor para que a construção seja bem sucedida e o instrumento possa operar como esperado.

Portanto, baseado na importância histórica, e na capacidade operacional do instrumento em questão, podemos afirmar que é possível incorporá-lo em uma proposta de interface entre história e ensino de matemática, ressaltando os potenciais que estão presentes, tanto no procedimento de construção aqui exposto, como na sua eventual utilização para a operação de multiplicação e divisão.

\footnotetext{
${ }^{17}$ Em inglês, lê-se: "one of the digits should be written or carved in the larger margin of each strip" (NAPIER, 2017, p. 714)
} 
Pedro Henrique Sales Ribeiro, Davi Souza Cavalcante, Ana Carolina Costa Pereira

O procedimento de construção do Promptuario de John Napier (1550-1617)

\section{Referências}

BRASIL. Base Nacional Comum Curricular. Brasília: MEC, 2018. Disponível em: http://basenacionalcomum.mec.gov.br/abase/. Acesso em: 16 set. 2020

KRIPKA, R. M. L.; SCHELlER, M. e BONOTTO, D. L. Pesquisa Documental: considerações sobre conceitos e características na Pesquisa Qualitativa. IN: CONGRESSO IBERO-AMERICANO EM INVESTIGAÇÃO QUALITATIVA, 4., 2015, Aracaju. Anais. Aracaju: Ludomedia. 2015. p. 243-247.

MARTINS, E.; PEREIRA, A. C. C. Uma primeira descrição da obra: Rabdologiae, seu numerationis per virgula ... DE 1617. Boletim Cearense de Educação e História da Matemática, v. 5, n. 14, p. 154 - 166, 25 ago. 2018.

MARTINS, E.; PEREIRA, A. C. C. AS BARRAS DE CALCULAR DE NAPIER: Percepções de uma primeira manipulação. Boletim Cearense de Educação e História da Matemática, v. 6, n. 18, p. 53 - 64, 31 dez. 2019.

NAPIER, J. Rabdologiae, Seu Numerationis Per Virgulas: cum appendice de expeditíssimo Multiplicationes promptuario, quibus acessit e arithmeticea localis liber unus. In: RICE, B.; GONZÁLEZ-VELASCO, E.; CORRIGAN, A. The Life and Works of John Napier. Cham: Springer, 2017. p. 652-749.

RICE, B.; GONZÁLEZ-VELASCO, E.; CORRIGAN, A. The Life and Works of John Napier. Cham: Springer, 2017.

SAITO, F. História da matemática e suas (re)construções contextuais. São Paulo: Editora Livraria da Física, 2015. p. 259.

SILVA, I. C. Um estudo da incorporação de textos originais para a educação matemática: buscando critérios na articulação entre história e ensino. 2018. 92 f. Dissertação (Mestrado) - Curso de Programa de Pós-Graduação em Ensino de Ciências e Matemática - PGECM - Instituto Federal de Educação, Ciência e Tecnologia do Ceará, Fortaleza, 2018. Disponível em: http://pgecm.fortaleza.ifce.edu.br/wpcontent/uploads/2018/11/Dissertação-Isabelle-Coelho-da-Silva.pdf. Acesso em $02 \mathrm{dez}$. 2020.

Recebido em: 07 /12 / 2020

Aprovado em: 09 / 12 / 2020 